Chirurg 2014 · 85:1014

DOI 10.1007/s00104-014-2902-z

Online publiziert: 12. November 2014

C Springer-Verlag Berlin Heidelberg 2014

O. Strobel · M.W. Büchler

Klinik für Allgemein-, Viszeral- und Transplantationschirurgie, Universität Heidelberg

\title{
Borderline-Resektabilität beim Pankreaskarzinom: Konsensus-Statement der ISGPS
}

- Besprechung in einem Tumorboard an einem High-volume-Zentrum.

- Kriterien sind: Einengung oder Verschluss der Pfortader/Vena mesenterica superior (VMS) mit anschlussfähigen Gefäßen, Umschließen der A. gastroduodenalis oder kurzstreckiges Umschließen der A. hepatica, aber nicht des Truncus coeliacus, Erreichen der A. mesenterica superior mit Kontakt $\leq 180 \%$ der Zirkumferenz.

Mit der stetigen Verbesserung der Ergebnisse in der Pankreaschirurgie werden zunehmend schwierigere Resektionen durchgeführt. Früher als nicht resektabel geltende Tumoren werden heute häufig reseziert. Die International Study Group of Pancreatic Surgery (ISGPS) hat nun Konsensus-Statements publiziert, die durch einheitliche Definitionen künftige Untersuchungen zu erweiterter Pankreasresektionen erleichtern und andererseits klinische Handlungsempfehlungen beinhalten. Neben dem Konsensus-Statement zur Boderline-Resektabilität wurden aktuell Statements zu multiviszeralen erweiterten Pankreasresektionen [1] und zur standardisierten Lymphadenektomie [2] beim Pankreaskarzinom veröffentlicht.

\section{Methoden und Ergebnisse}

Ein internationales Panel von Pankreaschirurgen aus etablierten Zentren für Pankreaschirurgie führte eine systematische Literaturanalyse durch und entwickelte auf dieser Basis die folgenden KonsensusStatements und Empfehlungen.

- Kriterien der Borderline-Resektabilität und deren Erhebung:

- Erhebung anhand einer höchstens 4 Wochen alten Computertomographie mit spezialisiertem Pankreasprotokoll.
- Indikation zur operativen Exploration und Resektion bei Beteiligung der Pfortader/VMS:

- Gute Evidenz für primäre Resektion bei rekonstruktionsfähiger venöser Infiltration.

- Keine Evidenz für neoadjuvante Therapie bei isolierter venöser Beteiligung.

- Intraoperative Entscheidung zur Resektion mit venöser Rekonstruktion, wenn komplette Resektion möglich erscheint.

- Venöse Resektionen sollten in Zentren durchgeführt werden.

- Die Art der venösen Resektion sollte klassifiziert und berichtet werden.

- Indikation zur operativen Exploration und Resektion bei Anhalt für arterielle Infiltration:

- Arterielle Resektion wird bei Pankreaskopfkarzinom mangels Evidenz nicht empfohlen.

- Chirurgische Exploration zur Klärung einer arteriellen Infiltration bei Borderline-Kriterien.

- Bei arterieller Infiltration ist eine Palliativtherapie Standard. Neoadjuvante Therapiekonzepte und nicht kurative Resektionen sollten nur innerhalb klinischer Studien durchgeführt werden.
- Pathologische Befundung:

- Die verwendete R1-Definition (Tumorzellen am Schnittrand vs. $\leq 1 \mathrm{~mm}$ vom Schnittrand) sollte genannt werden.

- Untersuchung aller sieben Absetzungsränder.

- Bei Gefäßresektion detaillierte Untersuchung der Gefäßinvasion.

\section{Fazit}

Anhand der aktuellen Datenlage kann bei Patienten mit Borderline-resektablem Pankreaskarzinom eine primäre Operation mit venöser Resektion empfohlen werden. Diese anspruchsvollen Resektionen sollten in entsprechend spezialisierten Zentren durchgeführt werden.

\section{Korrespondenzadresse}

\section{PD Dr. 0. Strobel}

Klinik für Allgemein-, Viszeralund Transplantationschirurgie, Universität Heidelberg, Im Neuenheimer Feld 110, 69120 Heidelberg

Oliver.Strobel@med.uni-heidelberg.de

Interessenkonflikt. O. Strobel und M.W. Büchler geben an, dass kein Interessenkonflikt besteht.

\section{Literatur}

1. Hartwig W, Vollmer CM, Fingerhut A et al (2014) Extended pancreatectomy in pancreatic ductal adenocarcinoma: definition and consensus of the International Study Group for Pancreatic Surgery (ISGPS). Surgery 156:1-14

2. Tol JA, Gouma DJ, Bassi C et al (2014) Definition of a standard lymphadenectomy in surgery for pancreatic ductal adenocarcinoma: a consensus statement by the International Study Group on Pancreatic Surgery (ISGPS). Surgery (Epub ahead of print) PMID: 25061003 